\title{
Educación médica durante la pandemia. ¿Qué médicos preparamos?
}

\author{
Medical education during the pandemic: which physicians do we prepare? \\ Federico L Rodríguez Weber,* José Luis Ramírez Arias ${ }^{\ddagger}$ \\ Citar como: Rodríguez WFL, Ramírez AJL. Educación médica durante la pandemia. ¿Qué médicos preparamos? \\ Acta Med Grupo Angeles. 2021; 19 (3): 325-326. https://dx.doi.org/10.35366/101722
}

Sin duda, la pandemia ha tenido una repercusión mundial y ha alcanzado prácticamente todas las esferas de las actividades humanas, entre otras a la de la Educación Médica; conforme ha avanzado la enfermedad hemos tenido que aprender a cambiar muchas cosas en relación al diagnóstico, la fisiopatología, el tratamiento, los apoyos, la rehabilitación y la prevención, así como el agente causal y sus diferentes mutaciones, también hemos aprendido a fortalecer y promover las medidas de protección sanitaria.

Desde el inicio de la pandemia todos los gobiernos dictaron medidas para contener en lo posible a la enfermedad, desde el uso de cubrebocas, lavado de manos, uso de gel antiséptico hasta el distanciamiento y confinamiento, obligando a que todas las actividades humanas se vieran afectadas, una de éstas fue el impacto en la educación formal en todos los niveles.

A la fecha, en casi en todos los países, se trata de fomentar una política de regreso a las actividades presenciales, principalmente en el Sector de Educación. En México llama la atención que aún continúan llevándose a cabo muchos cursos de educación superior a distancia, los cuales tratan de compensar parcialmente la pérdida de elementos que proporciona la educación presencial. Lo anterior sucede cuando la mayoría de los profesores y alumnos ya están vacunados y están conscientes de las medidas obligatorias de protección.

No es la excepción el trabajo en las escuelas, facultades de medicina e instituciones de salud que tienen inicialmente el reto de mantener funcionando los diferentes cursos y programas, además de dar apoyo a la atención de pacientes con COVID-19. A nivel de licenciatura se ha migrado mediante las tecnologías de la información y comunicación (TIC) a mantener los cursos a distancia, abandonando, o al menos disminuyendo, la práctica en los hospitales y sitios de atención clínica. ${ }^{1}$ Esta situación que se ha prolongado hasta el momento actual, no nos permite saber si lo realizado ha sido suficiente para formar adecuadamente a los médicos y si éstos desarrollarán las habilidades necesarias para poder ejercer en forma apropiada la medicina.

Ante esta situación surgen muchas preguntas como: ¿es necesario que los futuros médicos tengan conciencia y aprendizaje de las medidas de protección necesarias que se deben tomar en el ejercicio profesional y conocer los riesgos que implica la práctica médica?, ¿quedará claro que eso es parte de la práctica clínica de los médicos? No sabemos si estamos dejando de lado la oportunidad del aprendizaje en la forma práctica, debido a los pacientes contagiosos que siempre existirán en pandemia o sin pandemia y los riesgos que siempre existen, por lo que se invita a las autoridades a preguntarse: ¿no deberían los estudiantes de medicina en todos sus niveles estar familiarizándose con esta nueva forma de atender pacientes aprendiendo a tomar las medidas preventivas y de seguridad necesarias, asumiendo que hay riesgos en los trabajadores de la salud a las que se enfrentan cotidianamente?; ¿se está limitando la formación de los futuros médicos?; ¿no será que estamos formando médicos que en el futuro, en situaciones de riesgo, querrán manejar todo a distancia o se prefieran "esconder" en aislamiento por el riesgo de contagio?

\footnotetext{
Correspondencia:

Dr. Federico L Rodríguez Weber

Correo electrónico: fweber@saludangeles.com
}

la Universidad La Salle. Coordinador de pregrado y postgrado Grupo Ángeles Servicios de Salud (GASS).

₹ Director Médico del Hospital Ángeles Pedregal; Excoordinador del PUEM de Radiología de la FACMED UNAM, Academia Nacional de Medicina. www.medigraphic.com/actamedica

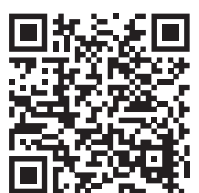


En los estudios de postgrado la posición resulta un poco diferente, pues la situación dependerá de la especialidad de que se trate y de la institución involucrada en la formación de los especialistas, la limitación de su formación podrá ser mayor o menor de acuerdo con la experiencia adquirida durante este tiempo; sólo por citar algunos ejemplos: los cirujanos plásticos seguramente en este periodo disminuyeron su trabajo, sobre todo en instituciones en donde el presupuesto y la labor asistencial fue reasignada para la atención prioritaria de pacientes COVID; otro ejemplo es la especialidad de cardiología en donde durante la pandemia solamente se atendía lo más urgente, también por dar prioridad a los pacientes infectados con COVID; esto condujo a que muchas enfermedades avanzaran, empeorando el pronóstico y agravándose, por lo que tenían que atenderse como emergencias médicas. Lo anterior se podría transpolar a la gran mayoría de especialidades, ya que muy pocas instituciones continuaron la atención de todos los pacientes con necesidades médicas diferentes a COVID, pero la formación de especialistas continuó sus programas en todas las especialidades sin poder garantizar si la experiencia académica, asistencial y de destrezas fue suficiente. ${ }^{2,3}$

Una situación intermedia es la que se presenta en el internado de pregrado por ser el último año formal de la formación del médico y en el que los alumnos se pasan un año en el hospital rotando por los servicios y adquiriendo el conocimiento y las destrezas para poder ejercer posteriormente; ${ }^{4}$ a este grupo también se les quitó, por lo menos por algún tiempo, la oportunidad de adquirir conocimientos y destrezas, lo que seguramente habrá generado carencias en el proceso de formación y en la adquisición de conocimientos $y$, por ende, seguramente se estarán egresando médicos que de alguna manera estarán incompletos en su formación, o por lo menos tendrán un perfil diferente al pactado con la sociedad.
Los ajustes provocados por la pandemia dan la impresión de que será necesario formar profesionales de la salud con menor compromiso con los pacientes. En un modelo en el que la tecnología y la atención médica a la distancia será el común denominador de la práctica médica y en donde el contacto con el paciente no existe o está limitado, entre otros, por el riesgo o miedo de adquirir una enfermedad contagiosa a pesar de que se cuenten con las medidas de protección y prevención al alcance de los profesionales que proporcionan la atención médica. Lo anterior afecta a los aspectos cognitivos y las destrezas en el médico y en el especialista en formación, creemos que es momento de preguntarnos: iestamos formando los médicos con el perfil que deseamos? Si la respuesta es no, es necesario pensar cómo retomar el camino para formar médicos sin limitaciones utilizando todos los beneficios de la tecnología en beneficio propio y de sus pacientes, sin alejarnos de la esencia misma de la medicina que es el paciente, haciendo de esta actividad profesional una actividad esencialmente de contacto humano en la que se reconoce la importancia de la relación profesional médico-paciente.

\section{REFERENCIAS}

1. Eva KW, Brownie AM. Medical education adaptations: really good stuff for educational transition during a pandemic. Med Educ. 2020; 54 (6): 494-494. doi: 10.1111/medu.14172.

2. Gallo G, Trompetto M. The effects of COVID-19 on Academic Activities and Surgical Education in Italy. J Invest Surg. 2020; 33 (7): 687-689. doi: 10.1080/08941939.2020.1748147.

3. Ehrlich $\mathrm{H}$, McKenney M, Elkbuli A. We asked the experts: virtual learning in surgical education during the COVID-19 pandemicshaping the future of surgical education and training. World J Surg. 2020; 44 (7): 2053-2055. doi: 10.1007/s00268-020-05574-3.

4. Ríos-Cortázar V, Gasca-García A, Urbina-García R, Flores-Echavarría $\mathrm{R}$, Lloret-Rivas A. Nuevos modelos educativos en el internado médico de pregrado. La participación de la Universidad. Reencuentro. 2005; 42: 1-16. 